Mindestlöhne nach dem Arbeitnehmerentsendegesetz, in: Knickrehm/Rust (Hrsg.), Arbeitsmarktpolitik in der Krise. Festgabe für Karl-Jürgen Bieback, Baden - Baden 2010, S. 185 201, ISBN 978-3-8329-5076-7

\title{
Andreas Hänlein
}

\section{Mindestlöhne nach dem Arbeitnehmerentsendegesetz}

\section{Einleitung}

Das Thema „Mindestlöhne und Arbeitnehmerentsendegesetz“ lässt sich nicht ohne weiteres unter das Generalthema „Reicht die Hartzgesetzgebung?“ subsumieren. Der Hartzgesetzgebung und dem Arbeitnehmerentsenderecht, zumal dem Entsenderecht in seiner heutigen Erscheinungsform liegen vielmehr nachgerade konträre politische Konzepte zugrunde. Um diese These zu belegen, wird hier zunächst die Entwicklung des AEntG nachgezeichnet: vom hochumstrittenen AEntG 1996 der Regierung Kohl/Genscher bis zum AEntG 2009 der Großen Koalition (II). Das AEntG 2009 ist geprägt von einer neuen Mindestlohnpolitik und überdies auch von der Rechtsprechung des EuGH zur Dienstleistungsfreiheit. Vor diesem Hintergrund geht das neue Recht einher mit gravierenden Eingriffen in die Tarifautonomie. Vom Streit, der um diese Eingriffe bereits im Vorfeld des neuen Gesetzes entstanden ist, soll ebenfalls die Rede sein (III). Hat sich das AEntG 2009 als Baustein einer neuen Mindestlohnpolitik erwiesen, lässt sich schließlich leicht zeigen, dass es dem Geist der Hartzgesetzgebung insofern widerspricht, als diese - mit dem SGB II - versucht hat, eine Niedriglohnpolitik zu realisieren (IV).

\section{Die Mutation des AEntG von der ,punktuellen Lösung“ zum weitreichenden Min- destlohngesetz}

\section{Die Ausgangslage}

Das im Kontext von Mindestlöhnen unmittelbar einschlägige Gesetz über die Festsetzung von Mindestarbeitsbedingungen aus dem Jahr $1952^{1}$ war in der Praxis nie angewendet worden ${ }^{2}$.

\footnotetext{
${ }^{1}$ Gesetz vom 11.1.1952, BGBl. I S. 17.

${ }^{2}$ Vgl. auch Thüsing, Mindestlohn im Spannungsfeld staatlicher und privatautonomer Regelung, ZfA 2008, 590, 592: ,dormant law“.
} 
Wo es sinnvoll erschien, Mindestarbeitsbedingungen allgemein durchzusetzen, wurde das Instrument des allgemeinverbindlichen Tarifvertrages eingesetzt. Dieses Instrument war insbesondere in der Bauwirtschaft von großer Bedeutung. Gerade in dieser Branche wurde die Wirkung des allgemeinverbindlichen Tarifvertrages Anfang der 90er Jahre jedoch partiell dadurch unterlaufen, dass damals zunehmend Bauarbeiter aus anderen Mitgliedstaaten der Europäischen Gemeinschaft auf deutschen Baustellen eingesetzt wurden, vorwiegend aus Portugal, Großbritannien, Irland und Griechenland. Auf der Grundlage von Abkommen mit den mittel-und osteuropäischen Reformstaaten waren überdies Arbeitnehmer aus diesen Staaten in spürbarem Umfang in Deutschland aktiv. All diese Arbeitnehmer waren bei im Ausland ansässigen Unternehmen beschäftigt und wurden von ihren Arbeitgebern nach Deutschland entsandt. Die mit den entsandten Arbeitnehmern geschlossenen Arbeitsverträge, die regelmäBig nicht deutschem Arbeitsvertragsrecht unterliegen, sahen deutlich schlechtere Arbeitsbedingungen, insbesondere niedrigere Löhne vor, als sie für die Kollegen galten, für deren Arbeitsverträge das deutsche Recht maßgeblich war ${ }^{3}$.

Diese Form der Arbeitsmigration hatte der Europäische Gerichtshof damals deutlich erleichtert. Er hatte 1990 entschieden, dass die Entsendung von Arbeitnehmern von der Dienstleistungsfreiheit der entsendenden Unternehmen erfasst sei ${ }^{4}$; im damaligen Ausgangsfall waren portugiesische Arbeitnehmer nach Frankreich entsandt worden, und zwar zu einer Zeit, als diese selbst sich wegen der nach dem Beitritt Portugals laufenden Übergangsfrist noch nicht auf die Freizügigkeit der Arbeitnehmer hatte berufen können. In einem Urteil aus dem Jahr 1994 entschied der Gerichtshof überdies, dass sich die Dienstleistungsfreiheit auch auf die Mitnahme von Personal aus Drittstaaten erstrecken kann ${ }^{5}$. In beiden Urteilen wies der Gerichtshof allerdings zugleich darauf hin, dass es das Gemeinschaftsrecht den Mitgliedstaaten nicht verwehre, ihre Rechtsvorschriften oder Tarifverträge über Mindestlöhne auf alle Personen auszudehnen, die in ihrem Hoheitsgebiet, und sei es auch nur vorübergehend, eine unselbständige Erwerbstätigkeit ausüben, und zwar unabhängig davon, in welchem Land der Arbeitgeber ansässig ist; ebensowenig verbiete das Gemeinschaftsrecht den Mitgliedstaaten, die Beachtung dieser Regeln mit den geeigneten Mitteln durchzusetzen ${ }^{6}$.

Die allgemeinverbindlichen Tarifverträge der Baubranche waren jedoch kein wirksames Instrument, mit dem den entsendenden Unternehmen der Lohnkostenvorteil hätte genommen

\footnotetext{
${ }^{3}$ Eine Situationsbeschreibung findet sich bei Koberski/Ashoff/Hold, AEntG, 2. Aufl., 2002, Einleitung, Rn. 2 5; vgl. auch die Begründung des Fraktionsentwurfs zum AEntG BT-Drs. 13/2414, S. 6 f.

${ }^{4}$ EuGH, 27.3.1990 - Rs. C-113/89 - (,Rush Portuguesa“).

${ }^{5}$ EuGH, 9.8.1994 - C 43/93 - (,Van der Elst“").

${ }^{6}$ EuGH, 27.3.1990 - Rs. C-113/89 - (,Rush Portuguesa“), Rn 18; 9.8.1994 - C-43/93 - (,Van der Elst“), Rn. 23.
} 
werden können, denn allgemeinverbindliche Tarifverträge konnten (und können) nach Auffassung des BAG auf Arbeitsverhältnisse nicht angewendet werden, die einem ausländischen Recht unterliegen; diese Rechtslage wurde auch nicht als unvereinbar mit dem „ordrepublic"-Vorbehalt des Art. 30 EGBGB a.F. angesehen ${ }^{7}$.

In dieser Situation forderte eine Koalition aus IG-Bau und Bauunternehmen die Einführung von Mindestlöhnen für nach Deutschland entsandte ausländische Bauarbeiter ${ }^{8}$. Die Bemühungen der Bundesregierung, auf europäischer Ebene eine Regelung herbeizuführen, scheiterten zunächst am Widerstand der Herkunftsstaaten der entsandten Arbeitnehmer (v.a. Portugal, Vereinigtes Königreich) ${ }^{9}$. Nach heftigem innenpolitischem Ringen gab schließlich die FDP ihren ursprünglichen Widerstand gegen eine nationale Regelung auf. Das Ergebnis war das Arbeitnehmerentsendegesetz ${ }^{10}$.

\section{Das AEntG der Kohl-Regierung: eine „punktuelle Lösung“6}

Das Arbeitnehmerentsendegesetz vom 26. Februar $1996^{11}$ sah vor, dass die Rechtsnormen eines für allgemeinverbindlich erklärten Tarifvertrages des Bauhaupt- und des Baunebengewerbes über ein Mindestentgelt sowie über die Dauer des Erholungsurlaubs, das Urlaubsentgelt oder ein zusätzliches Urlaubsgeld und auch über das Urlaubskassenverfahren auch auf die Arbeitsverhältnisse aus dem Ausland entsandter Arbeitnehmer „Zwingend“ Anwendung zu finden hatten ( $\$ 1$ AEntG 1996). Auf diese Weise wurden die betreffenden Rechtsnormen der allgemeinverbindlichen Tarifverträge des Baugewerbes auf Arbeitgeber mit Sitz im Ausland erstreckt und zugleich zu zwingenden Vorschriften im Sinne des Internationalen Privatrechts (Art. 34 EGBGB) erklärt. ${ }^{12}$

\footnotetext{
${ }^{7}$ BAG 4.5.1977 - 4 AZR 10/76 - E 29, 138 = AP Nr. 60 zu § 1 TVG Tarifverträge: Bau; 9.7.2003 - 10 AZR 593/02 -, AP Nr. 261 zu § 1 TVG Tarifverträge: Bau; in dieser Entscheidung stellt dass BAG fest, dass die Rechtsnormen allgemeinverbindlicher Tarifverträge keine Eingriffsnormen im Sinne von Art. 34 EGBGB darstellen; aus der Literatur: ErfK/Schlachter, 9. Aufl, 2009, § 1 AEntG Rn. 1 u 8 m.w.N; Hänlein, Die rechtliche Stellung der in Deutschland tätigen Werkvertragsarbeitnehmer aus der Türkei, ZIAS 1996, 21, 39 f.; kritisch Däubler in: Däubler(Hrsg.) TVG, 2. Aufl., 2006, Einleitung, Rn. 656.

${ }^{8}$ Vgl. z.B. FAZ v. 7.11.1995: „IG Bau kämpft weiter für das Entsendegesetz“; zur Position des DGB Kehrmann/Spirolke, Entwurf eines Arbeitnehmer-Entsendegesetzes für die Bauwirtschaft, AiB 1995, 621.

${ }^{9}$ Zur Vorgeschichte der Entsende-Richtlinie 96/71/EG Koberski/Asshoff/Hold, AEntG 2. Aufl, 2002, Enleitung Rn 17).

${ }^{10}$ Übersicht zum Gesetzgebungsverfahren bei Koberski/Asshoff/Hold, AEntG, 2. Aufl., 2002, Einleitung, Rn 1; zu den Veränderungen des Gesetzes im Laufe des Gesetzgebungsverfahrens Sahl/Stang, AiB 1996, S. 652 (S. 654 f.)

${ }^{11}$ BGBl. I 227.

${ }^{12}$ So ausdrücklich der Regierungsentwurf BT-Drs. 13/2414, 8; ebenso die spätere Rechtsprechung des BAG; vgl. BAG 14.8.2007 - 9 AZR 167/07 - NZA 2008, 236, 237.
} 
Dieses Gesetz war ausweislich der Gesetzesbegründung in mehrfacher Hinsicht als eine „,punktuelle Lösung“" konzipiert ${ }^{13}$. Es beschränkte sich zunächst auf eine bestimmte Branche, nämlich auf die Bauwirtschaft. Sodann bezog es sich ausschließlich auf bestimmte, in allgemeinverbindlichen Tarifverträgen geregelte Arbeitsbedingungen, nämlich auf Lohn und Urlaub. Hinsichtlich des Lohnes kam eine Erstreckung der betreffenden Normen nur in Frage, soweit der Tarifvertrag ,ein für alle unter seinen Geltungsbereich fallenden Arbeitnehmer einheitliches Mindestentgelt" enthielt. Schießlich wurde die Geltung des Gesetzes befristet. Es sollte am 1.9.1999, also dreieinhalb Jahre nach seinem Inkrafttreten, wieder außer Kraft treten; die Befristung wurde mit der Erwägung begründet, dass sich die unterschiedlichen Lohnniveaus in den verschiedenen Mitgliedstaaten angleichen würden, so dass sich ein Sondergesetz für die Bauwirtschaft nur für einen überschaubaren Zeitraum rechtfertigen lasse ${ }^{14}$. Das Gesetz sah überdies Kontrollmechanismen vor; vor allem hinsichtlich der Kontrollverfahren hatte die SPD-Mehrheit im Vermittlungsausschuss Verschärfungen durchsetzen können ${ }^{15}$.

\section{Rot-grüne Korrekturen des AEntG 1998: Abkehr von der „punktuellen Lösung““}

Nachdem bereits in der 13. Legislaturperiode aufgrund der ersten Erfahrungen mit dem Gesetz einige Änderungen vorgenommen worden waren ${ }^{16}$, veränderte das sog. Korrekturgesetz der ersten rot-grünen Koalition den Charakter des AEntG entscheidend ${ }^{17}$. Von einer ,punktuellen Lösung“ kann seitdem nicht mehr die Rede sein. Die durch dieses Gesetz vorgenommenen Änderungen des AEntG dienten zum einen der Umsetzung der in der Zwischenzeit in Kraft getretenen Entsende-Richtlinie ${ }^{18}$, der die Mitgliedstaaten seit dem 16.12.1999 nachzukommen hatten. Vor diesem Hintergrund wurde die Befristung des Gesetzes beseitigt ${ }^{19}$. Der Umsetzung des Konzepts der Richtlinie diente auch die Ausdehnung des Anwendungsbereichs des AEntG auf weitere Aspekte der Arbeitsbedingungen (Arbeitszeit; Arbeitssicherheit; Arbeitsschutz und Gleichbehandlung; dazu $§ 7$ AEntG a.F. sowie Art. 3 Abs. 1 RL

\footnotetext{
${ }^{13}$ BT-Drs. 13/2414, 7 ff.

${ }^{14}$ So BT-Drs. 13/2414, 8 zur zunächst geplanten zweijährigen Befristung.

${ }^{15}$ Vgl. Beschlussempfehlung des Vermittlungsausschusses v. 1.2.1996, BT-Drs. 13/3663; dazu Sahl/Stang, AiB $1996,652,655$.

${ }^{16}$ Durch das 1. SGB III-Änderungsgesetz v. 16.12.1997, BGB1. I., 2970; Entwurf BT-Drs. 13/8012; Übersicht über die Änderungen bei Koberski/Asshoff/Hold, AEntG, 2. Aufl., 2002, Einleitung, Rn. 39 - 46.

${ }^{17}$ Art. 10 des Gesetzes zu Korrekturen in der Sozialversicherung und zur Sicherung der Arbeitnehmerrechte v. 19.12.1998, BGB1. I, 3843, Entwurf BT-Drs. 14/45.

${ }^{18}$ Richtlinie 96/71/EG des Europäischen Parlaments und des Rates vom 16.Dezember 1996 über die Entsendung von Arbeitnehmern im Rahmen der Erbringung von Dienstleistungen, ABl. 1997 Nr. L 18 S. 1; wegen verschiedener Umsetzungsdefizite leitete die Kommission ein Vertragsverletzungsverfahren gegen Deutschland ein, das mit einem Teilerfolg beim EuGH endete; vgl. EuGH 18.7.2007, - Rs. C-490/04 - (Kommission./. BRD), ZESAR 2007, S. 487 ff. m. Anm. Hänlein, ZESAR 2007, 494.

${ }^{19}$ BT-Drs. 14/ 45, 17.
} 
96/71/EG). Insoweit handelt es sich allerdings nicht um eine Änderung von großer Tragweite, da die betroffenen Regelungen ohnehin als „,zwingende“ Bestimmungen im Sinne des Art. 34 EGBGB angesehen werden ${ }^{20}$.

Die meisten weiteren Änderungen sind mit dem geänderten politischen Klima nach dem Regierungswechsel im Herbst 1998 zu erklären. Sie erfolgten „,im Interesse einer wirksamen

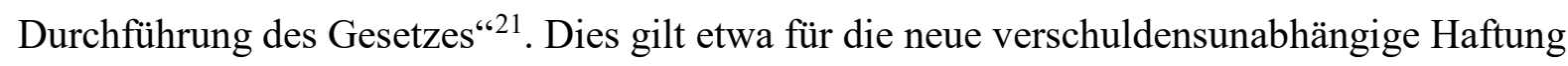
des Generalunternehmers ( $§ 1 \mathrm{a}$ AEntG 1999) und für die Erweiterung der Kontrollmöglichkeiten. Des weiteren wurde den Tarifpartnern die Möglichkeit eröffnet, nach dem Entsendegesetz zu verallgemeinernde Mindestlöhne nicht nur für eine unterste Lohngruppe, sondern auch für höhere Lohngruppen vorzusehen ${ }^{22}$.

Zwei Änderungen sind für den Wandel des Gesetzes zu einem auch inlandsbezogenen Instrument zur Durchsetzung von Mindestlöhnen besonders bedeutsam.

Die erste dieser Änderungen betrifft die Tragweite der zwingenden Wirkung, die das AEntG einem allgemeinverbindlichen Tarifvertrag verleiht. Allgemeinverbindliche Mindestlohntarifverträge waren bereits nach der ursprünglichen Fassung des AEntG ausdrücklich auch für Arbeitgeber mit Sitz im Inland verbindlich (§ 1 Abs. 1 S. 4 AEntG 1996). Um eine gemeinschaftsrechtlich unzulässige Diskriminierung ausländischer Arbeitgeber zu vermeiden, sollten diese nur solchen Tarifnormen unterworfen werden, die auch von inländischen Arbeitgebern verbindlich einzuhalten waren ${ }^{23}$. Da einige Gerichte jedoch die ordnungswidrigkeitenrechtliche Sanktionierung inländischer Arbeitgeber, die sich daran nicht hielten, nicht akzeptiert hatten $^{24}$, stellte der Gesetzgeber nun klar, dass die zwingende Wirkung eines allgemeinverbindlichen Tarifvertrages im Sinne des AEntG auch für einen Arbeitgeber mit Sitz im Inland gilt, und zwar ,unabhängig davon, ob der Tarifvertrag kraft Tarifbindung nach $\S 3$ des Tarifvertragsgesetzes oder aufgrund der Allgemeinverbindlicherklärung Anwendung findet" ( $§ 1$ Abs. 1 S. 3 AEntG 1999²5). Diese neue Formulierung hat das BAG später in einer Weise ausgelegt, die darauf hinausläuft, dass es nun im Ergebnis für das Entsenderecht einen allgemeinverbindlichen Tarifvertrag neuen Typs gibt. Die neue Version des Gesetzestexts schließt nach der Auslegung des BAG ,den Vorrang speziellerer Tarifverträge unabhängig davon aus, ob diese Tarifverträge darauf gerichtet sind, die Wirkungen der Erstreckung von Tarifverträ-

\footnotetext{
${ }^{20}$ So BT-Drs. 14/45, 27 zu Nr. 7.

${ }^{21}$ BT-Drs. 14/45, 17.

22 BT-Drs. 14/ 45, 17 u. 25.

${ }^{23}$ BT-Drs. 13/2414, 8.

${ }^{24}$ Koberski/Asshoff/Hold, AEntG, 2. Aufl., 2002, § 1 Rn. 6 m.w.N.; auch nach der viel später geäußerten Auffassung des BAG hatten unter Geltung des AEntG 1996 speziellere Tarifverträge Vorrang gegenüber allgemeinverbindlichen Tarifverträgen im Sinne des AEntG: BAG 20.7.2004 - 9 AZR 343/03 - E 111, 247, 254 f. = NZA $2005,114,117$.

${ }^{25}$ Mit AEntG 1999 ist hier und im folgenden das AEntG in der Fassung des Korrekturgesetzes gemeint.
} 
gen nach dem AEntG auszuschließen“26. Hintergrund dieser Auslegung war das Urteil des EuGH in der Rechtssache Portugaia Construcoes aus dem Jahr 2002. Der EuGH hatte damals eine unzulässige Diskriminierung von Arbeitgebern mit Sitz in anderen Mitgliedsstaaten für den Fall angenommen, dass sich zwar inländische, nicht jedoch ausländische Arbeitgeber der Bindung an erstreckte Tarifverträge durch speziellere tarifliche Regelungen entziehen können ${ }^{27}$. Im Bereich des Entsenderechts bewirkt ein allgemeinverbindlicher Tarifvertrag seitdem also nicht mehr nur eine Einbeziehung von Außenseitern, er kann vielmehr auch den Vorrang anderer Tarifverträge, die einen niedrigeren Mindestlohn vorsehen, beseitigen.

Von besonderer Bedeutung war ferner die durch das Korrekturgesetz eingeführte Möglichkeit, die Wirkung eines Tarifvertrages durch Rechtsverordnung auszudehnen, ohne dass die Voraussetzungen für eine Allgemeinverbindlicherklärung gegeben sein müssen; durch eine solche „Mindestlohnverordnung“ kann bestimmt werden, „dass die Rechtsnormen dieses Tarifvertrages auf alle unter den Geltungsbereich dieses Tarifvertrages fallenden und nicht tarifgebundenen Arbeitgeber und Arbeitnehmer Anwendung finden“ (§ 1 Abs. 3a S. 1 AEntG 1999). Für den Erlass einer solchen Rechtsverordnung durch das Bundesministerium für Arbeit und Sozialordnung genügte, dass ein entsprechender Antrag gestellt wurde; ein Einvernehmen mit dem Tarifausschuss, wie es $\S 5$ Abs. 1 TVG erfordert, war nicht erforderlich. Auch auf die materiellen Voraussetzungen dieser Vorschrift kam es nicht an. Der Hintergrund dieses neuen Instruments ministerieller Geltungserstreckung waren die Schwierigkeiten, die das Ministerium nach dem Inkrafttreten des AEntG bei der beabsichtigten Allgemeinverbindlicherklärung der ersten Mindestlohntarifverträge des Baugewerbes zu überwinden hatte ${ }^{28}$. Die diesbezügliche Allgemeinverbindlicherklärung wurde wegen des Widerstandes der Arbeitgeber aus anderen Branchen erst am 1.1.1997 wirksam, so dass das AEntG zunächst einmal für 10 Monate ohne praktische Auswirkung geblieben war.

Die Beschränkung der von der Rechtsverordnung ausgedehnten Tarifnormen auf „,nicht tarifgebundene Arbeitgeber und Arbeitnehmer“ erfolgte bewusst -, und zwar ausdrücklich ,mit Rücksicht auf die Tarifautonomie“ ${ }^{\text {29 }}$. Eine Kammer des BVerfG entschied im Juli 2000, dass die Verordnungsermächtigung mit dem Grundgesetz vereinbar sei; die in die Bindung eines

\footnotetext{
${ }^{26}$ BAG 26.2.2002 - 9 AZR 405/00 -; BAG 20.7.2004 - 9 AZR 343/03 - E 111, 247, 255 f. = NZA 2005, 114, 117; außerdem: BAG 9.9.2003 - 9 AZR 478/02 - (Anfragebeschluss); 13.5.2004 - 10 AS 6/04 - (Antwort); vgl. auch Lakies, in Däubler (Hrsg.)., TVG, 2. Aufl. 2002, § 5 Anhang 2, Rn. 107.

${ }^{27}$ EuGH 24.1.2002, Rs. C-164/99 - (Portugaia Construções), Rn. 34.

${ }^{28}$ Näher Koberski/Asshoff/Hold, AEntG 2. Aufl, 2002, Einleitung, Rn. 29 - 33; vgl. auch Sahl/Stang, AiB 1996, $652,656$.

${ }^{29}$ BT-Drs. 14/45, 25.
} 
Tarifvertrages einbezogenen Außenseiter würden weder in ihrem Grundrecht auf positive noch auf negative Koalitionsfreiheit verletzt ${ }^{30}$.

Die Beschränkung der Verordnungsermächtigung auf nicht tarifgebundene Arbeitgeber und Arbeitnehmer versuchte das Bundesministerium für Arbeit und Soziales Ende 2007 zu ignorieren, als es die Post-Mindestlohnverordnung ${ }^{31}$ erließ, wenige Tage, nachdem die Briefdienstleistungen in das AEntG aufgenommen worden waren. Mit dieser auf $\S 1$ Abs. 3a AEntG 1999 gestützten Verordnung sollten die Mindestlöhne, die der Arbeitgeberverband Postdienste e.V. mit der Gewerkschaft ver.di für Briefzusteller vereinbart hatte, auf alle nicht an diesen Tarifvertrag gebundenen Arbeitgeber und Anwendung erstreckt werden. Der Sinn der Regelung bestand darin, die vom Arbeitgeberverband der Konkurrenten der Post mit der Gewerkschaft der neuen Brief- und Zustelldienste vereinbarten niedrigeren Mindestlöhne zu überspielen. In dem verwaltungsgerichtlichen Verfahren, in dem die Post-

Mindestlohnverordnung als rechtswidrig angegriffen worden war, verlor die beklagte Bundesrepublik Deutschland, da die Verordndung von der Ermächtigungsgrundlage nicht gedeckt war, die eben nur Verordnungen erlaubte, die nicht tarifgebundene Arbeitgeber und Arbeitnehmer in einen erstreckten Tarifvertrag einbeziehen ${ }^{32}$.

\section{Marginale Änderungen durch die Hartz-Gesetze}

Die Hartz-Gesetzgebung hat nur wenige Änderungen des AEntG mit sich gebracht. Eine gewisse Ausweitung enthält allein das Erste Gesetz für moderne Dienstleistungen am Arbeitsmarkt vom 23.12.2002 ${ }^{33}$, das den Anwendungsbereich des AEntG auf Leiharbeitnehmer erweiterte, die von einem Entleiher in der Baubranche eingesetzt werden.

Allerdings entwickelte sich gegen Ende der zweiten Schröderregierung eine lebhafte politische Debatte um die Forderung nach einer Einführung von Mindestlöhnen ${ }^{34}$. Diese Diskussion lässt sich als Reaktion auf das Konzept der „Hartzgesetze“ verstehen ${ }^{35}$ und zugleich auf eine seit einigen Jahren zu beobachtende Erosion der Tarifbindung ${ }^{36}$.

\footnotetext{
${ }^{30}$ BVerfG 18.7.2000 - 1 BvR 948/00 -, NJW 2000, 3704.

${ }^{31}$ v. 28.12.2007, BAnz. v. 29.12.2007, Nr. 242, 8410.

${ }^{32}$ VG Berlin 7.3.2008 - VG 4 A 439.07 - NZA 2008, 482; im Ergebnis bestätigt durch OVG Berlin-

Brandenburg, 18.12.2008 - 1 B 13.08 - ; kritisch Greiner, Das VG Berlin und der Post-Mindestlohn, DB 2008, 840 ff.; ErfK/Franzen, 600, § 5 TVG, Rn. 5; a.A. Zipperling, Zum Post-Mindestlohn, DB 2008, 1790.

${ }^{33}$ BGBl. I 2002, 4607 (Art. 6a); Begründung im Ausschussbericht BT-Drs. 15/91, 17.

${ }^{34}$ Dazu Lakies in: Däubler/Lakies, TVG, 2. Aufl., 2006, Anhang 1 zu § 5, Rn. 23c m.w.N.

${ }^{35}$ Deutlich etwa Bispinck/Schäfer/Schulten, Argumente für einen gesetzlichen Mindestlohn, WSI-Mitt., 2004, 575.

${ }^{36}$ Vgl. etwa Freter, Das Tarifvertragssystem in der Krise, Essen 1998, S. 48 ff. und 52 ff.;

Bispinck/Kirsch/Schäfer, Projekt Mindeststandards für Arbeits- und Einkommensbedingungen und Tarifsystem,
} 
Die damalige Bundesregierung griff die Forderung nach einem gesetzlichen Mindestlohn in Form eines Gesetzesvorschlags auf, der auf die Ausdehnung des AEntG auf alle Branchen abzielte $^{37}$. Vom Bundesrat wurde der Vorschlag jedoch abgelehnt. Er bedeute „de facto die Einführung eines gesetzlichen Mindestlohns durch die Hintertür, mit der Gefahr einer weiteren Steigerung der ohnehin schon zu hohen Arbeitskosten in Deutschland und daraus resultierend einer weiteren Verdrängung von Arbeitsplätzen gerade des Niedriglohnsektors ins Ausland“38. Zu einer Verabschiedung des Gesetzes kam es wegen des vorzeitigen Endes der Legislaturperiode nicht mehr.

\section{Weitreichende Mindestlohngesetzgebung der Großen Koalition}

In der Gesetzgebung der Großen Koalition wurde dann die Beschränkung des AEntG auf die Baubranche aufgegeben und damit der Charakter des Gesetzes nochmals gravierend verändert. Diese Entwicklung vollzog sich in mehreren Schritten.

Zunächst wurde die Ankündigung aus dem Koalitionsvertrag umgesetzt, auch das Gebäudereinigerhandwerk in das AEntG einzubeziehen. Dies war ein wichtiges Element des Ersten Änderungsgesetzes zum AEntG v. 25.4.2007 ${ }^{39}$. Ein zweiter Gesetzgebungsakt erstreckte das Gesetz - angesichts des Endes des Briefmonopols der Post - auf Briefdienstleistungen. Dies ist der Inhalt des 2. Änderungsgesetzes zum AEntG v. 21.12.2007 ${ }^{40}$. Die unmittelbar danach für die Briefdienstleistungen erlassene Mindestlohnverordnung ${ }^{41}$ war Gegenstand des bereits erwähnten Rechtsstreits beim VG Berlin.

Die umfangreichste Ausdehnung erfolgte schließlich durch das das „Gesetz über zwingende Arbeitbedingungen für grenzüberschreitend entsandte und für regelmäßig im Inland beschäftigte Arbeitnehmer und Arbeitnehmerinnen (Arbeitnehmer-Entsendegesetz) “ vom 20.4.2009 ${ }^{42}$. Dieses Gesetz war Bestandteil einer umfassenderen Mindestlohngesetzgebung, mit der das MiArbG geändert und das AEntG neu gefasst wurde ${ }^{43}$. Beim modernisierten MiArbG, das künftig nur noch Mindestvorgaben hinsichtlich des Entgelts ermöglicht, geht es nach wie vor darum, Mindestarbeitsbedingungen durch staatliche Regelungen festzulegen,

Projektbericht, 2003, S. 28 - 37; vgl. auch Lakies in: Däubler (Hrsg.), TVG, 2. Aufl., 2002, Anhang 1 zu § 5, Rn. $19 \mathrm{f}$.

${ }^{37}$ Gesetzesentwurf von SPD und Bündnis 90/Die Grünen vom 10.5.2005, BT-Drs. 15/5445; gleichlautend der Regierungsentwurf BT-Drs. 15/5810 vom 22.6.2005.

${ }^{38}$ BT-Drs. 15/5810, 6.

${ }^{39}$ BGBl. I, 576 f.; dazu Regierungsentwurf BT-Drs. 16/3064 und Ausschussbericht BT-Drs. 16/4554.

${ }^{40}$ BGB1. I, 3140; dazu Regierungsentwurf BT-Drs. 16/6735 und Ausschussbericht BT-Drs. 16/7512.

${ }^{41}$ Verordnung des Bundesministeriums für Arbeit und Soziales über zwingende Arbeitsbedingungen für die Branche Briefdienstleistungen vom 28. 12. 2007, BAnz. v. 29.12.2007, Nr. 242 S. 8410.

${ }^{42}$ BGBl. 2009 I, 799; dazu Regierungsentwurf BT-Drs. 16/10486 und Ausschussbericht BT-Drs. 16/11669.

${ }^{43}$ Überblick bei Sittard, Neue Mindestlohngesetze in Deutschland, NZA 2009, 346. 
und zwar in solchen Wirtschaftszweigen, in denen es entweder keine Tarifverträge gibt oder in denen Tarifbindung nur für eine Minderheit von Arbeitnehmern und Arbeitgebern besteht $^{44}$. Das AEntG, das im Interesse seine kodifikatorischen Qualität insgesamt neu gefasst wurde, baut demgegenüber das Konzept „tarifgestützter Mindestlöhne“ ${ }^{\text {“45 }}$ weiter aus.

Der Wandel des Gesetzes zu einem weitgehend von Entsendungsfällen unabhängigen Instrument der Lohnregulierung wird bereits an seinem neuen $\S 1$ über die Zielsetzung deutlich. Als Ziel des Gesetzes wird angegeben „die Schaffung und Durchsetzung angemessener Mindestarbeitsbedingungen für grenzüberschreitend entsandte und für regelmäßig im Inland beschäftigte Arbeitnehmer und Arbeitnehmerinnen sowie die Gewährleistung fairer und funktionierender Wettbewerbsbedingungen“( $§ 1$ S. 1 AEntG 2009). Die Zielgruppen der aus dem Ausland entsandten und der inländischen Arbeitnehmer stehen hier also mit gleichem Gewicht nebeneinander. Weitere Ziele sind die Erhaltung sozialversicherungspflichtiger Beschäftigung und die Wahrung der Ordnungs- und Befriedungsfunktion der Tarifautonomie (§ 1 S. 2 AEntG 2009).

Wichtig ist weiter, dass nunmehr fünf weitere Branchen in den Anwendungsbereich des Gesetzes einbezogen wurden ( $§ 4$ Nrn. 4 - 8 AEntG 2009) ${ }^{46}$. Weiterhin einbezogen wurde die Pflegebranche, für die allerdings ein Sonderregime vorgesehen ist ( $\S 10-13$ AEntG 2009). Bei den neu in das Gesetz aufgenommen Branchen handelt es sich zum Teil auch um Wirtschaftszweige, in denen die Entsendung ausländischer Arbeitnehmer jedenfalls bislang keine Rolle spielt ${ }^{47}$.

Offenkundig vor dem Hintergrund der gerichtlichen Auseinandersetzungen um die PostMindestlohnverordnung regelt das Gesetz nunmehr eingehend und einheitlich die Wirkung allgemeinverbindlicher Tarifverträge im Sinne des AEntG und der Mindestlohnverordnungen. Die Mindestlohnverordnung soll nun vorsehen, dass ein durch sie in seiner Wirkung ausgedehnter Tarifvertrag ,auf alle ... nicht an ihn gebundenen Arbeitgeber sowie Arbeitnehmer und Arbeitnehmerinnen Anwendung“ findet (§ 7 Abs. 1 S. 1 AEntG 2009). Ausdrücklich wird nunmehr für beide Instrumente gesagt, dass der erstreckte Tarifvertrag ,,von einem Arbeitgeber auch dann einzuhalten (ist), wenn er nach $\S 3$ TVG oder kraft Allgemeinverbindlicherklärung nach $\S 5$ TVG an einen anderen Tarifvertrag gebunden ist“" (§ 8 Abs. 2 AEntG 2009). Der erstreckte Tarifvertrag verdrängt mithin konkurrierende Tarifverträge. Durch

\footnotetext{
${ }^{44}$ BT-Drs. 16/10484, 1.

${ }^{45}$ So der Titel einer Publikation von Bieback/Dieterich/Hanau/Kocher/Schäfer aus dem Jahr 2007.

${ }^{46}$ Sicherheitsdienstleistungen, Bergbauspezialarbeiten auf Steinkohlebergwerken, Wäschereidienstleistungen im Objektkundengeschäft, Abfallwirtschaft einschließlich Straßenreinigung und Winterdienst sowie Aus- und Weiterbildungsdienstleistungen nach SGB II oder SGB III.

${ }^{47}$ So unter Hinweis auf Aus- und Weiterbildungsleistungen die Stellungnahme der FDP im Rahmen der Beratungen des Ausschusses für Arbeit und Soziales, BT-Drs. 16/11669, 19 (vorl. Fassung).
} 
diese „Klarstellung“ soll eine „durchgängige Geltung der tarifvertraglichen Arbeitsbedingungen" auch für inländische Arbeitnehmer bewirkt und so der Vorgabe des EuGH in seinem Urteil zur Rechtssache Portugaia Construcoes Rechnung getragen werden; zugleich werde auf diese Weise sichergestellt, dass es sich bei den erstreckten tariflichen Regelungen um ,für allgemein verbindlich erklärte Tarifverträge“ im Sinne von Art. 3 Abs. 8 der Entsenderichtlinie handelt ${ }^{48}$.

Geändert wurden überdies die Vorschriften über das Verfahren beim Erlass einer Mindestlohnverordnung ( 7 AEntG 2009). Neu ist zunächst das Erfordernis eines ,gemeinsamen Antrages“ der Parteien des Tarifvertrages, dessen Wirkung im Verordnungswege ausgedehnt werden soll (§ 1 Abs. 1 S. 1 AEntG 2009) ${ }^{49}$. Bezieht sich ein solcher Antrag auf eine Branche, in der zuvor noch keine Mindestentgeltverordnung galt, muss zunächst der Tarifausschuss ( 5 Abs. 1 S. 1 TVG) damit befasst werden ( 7 Abs. 5 AEntG 2009).

Des Weiteren reagieren neue Verfahrensregeln darauf, dass die Mindestlohnverordnungen künftig konkurrierende tarifliche Regelungen verdrängen. Für den Fall, dass es in einer Branche konkurrierende Tarifverträge gibt, muss der Verordnungsgeber die Parteien der betroffenen Tarifverträge anhören ( $§ 7$ Abs. 4 AEntG 2009) und bei seiner Entscheidung neben den Gesetzeszielen „die Repräsentativität der jeweiligen Tarifverträge“ berücksichtigen; bei der Feststellung der Repräsentativität ist vorrangig abzustellen auf die Zahl der vom jeweiligen Tarifvertrag erfassten Arbeitnehmer sowie auf die Zahl der erfassten Mitglieder der vertragsschließenden Gewerkschaft ( $\$ 7$ Abs. 2 AEntG 2009). Wurde in einem Fall konkurrierender Tarifverträge überdies hinsichtlich mehrerer dieser Verträge eine Mindestlohnverordnung beantragt, wird der Verordnungsgeber auf eine besonders sorgfältige Abwägung der „,betroffenen Güter von Verfassungsrang“ und auf einen „,schonenden Ausgleich“ der ,widerstreitenden Grundrechtsinteressen“ verpflichtet (§ 7 Abs. 3 AEntG 2009).

\section{Das AEntG 2009 im Streit}

Wie das bisherige dient auch das neue AEntG der Umsetzung der Entsende-Richtlinie und damit der Realisierung der Dienstleistungsfreiheit. Die Anforderungen, die sich hieraus ergeben, haben zur Folge, dass die einfachgesetzliche Ausgestaltung des Entsenderechts mit der grundrechtlich garantierten Tarifautonomie zu kollidieren droht, und zwar nach dem neuen AEntG in noch deutlicherer Form als bereits bislang. Die daraus resultierende verfassungs-

\footnotetext{
${ }^{48}$ BT-Drs. 16/10486, 17 (vorl. Fassung).

${ }^{49}$ Das Erfordernis eines ,gemeinsamen“ Antrages geht auf eine Beschlussempfehlung des Ausschusses für Arbeit und Soziales zurück; vgl. BT-Drs. 16/11669 (vorl. Fassung), 11, sub 2.5.b zu $§ 7$.
} 
rechtliche Problematik ist bereits im Vorfeld des förmlichen Gesetzgebungsverfahrens intensiv diskutiert worden. Vorschläge aus dieser Diskussion haben offenkundig den Entwurf des neuen AEntG beeinflusst und finden sich auch in der geltenden Fassung des Gesetzes wieder. Im folgenden wird der angedeutete Diskussionsverlauf nachgezeichnet und kommentiert.

\section{Das Bieback/Kocher Gutachten: Rechtfertigung des Vorrangs tarifgestützter Min- destlöhne}

Im Jahr 2007 hat die Hans-Böckler-Stiftung einen Sammelband zum Thema „Tarifgestützte Mindestlöhne“" publiziert ${ }^{50}$. Im Zentrum des Bandes steht ein Gutachten von Bieback und Kocher zu den „Juristischen Fragen der gesetzlichen Festlegung eines Mindestentgelts durch erleichterte Allgemeinverbindlichkeit oder Verweis auf unterste Tarifentgelte ${ }^{\text {“51 }}$. Das Gutachten erörtert eingehend die verfassungsrechtlichen Fragen, die entsenderechtliche Mindestlohnverordnungen aufwerfen und die bei einer Ausdehnung des Instruments der entsenderechtlichen Mindestlohnverordnung auf weitere Branchen noch größeres Gewicht als zuvor erhalten. Der Band enthält überdies Rechtsgutachten von Dieterich und von Hanau zur Bewertung tarifgestützter Mindestlöhne bei Zeitarbeit ${ }^{52}$. Auch diese Gutachten befassen sich mit den Verfassungsfragen der Mindestlohnverordnungen nach dem AEntG.

Alle erwähnten Autoren sind sich einig, dass die Ausdehnung des persönlichen Geltungsbereichs eines Tarifvertrages einen Eingriff in die durch Art. 9 Abs. 3 GG geschützte kollektive Koalitionsfreiheit darstellt. Dabei wird die Intensität des Eingriffs als unterschiedlich gravierend eingeschätzt, je nach dem, um welche der verschiedenen denkbaren Betroffenen es geht. Vom jeweiligen Gewicht des Eingriffs wird das Ausmaß der Rechtfertigungsnotwendigkeiten abhängig gemacht ${ }^{53}$.

\footnotetext{
${ }^{50}$ Bieback/Dieterich/Hanau/Kocher/Schäfer, Tarifgestützte Mindestlöhne, Baden-Baden 2007.

${ }^{51}$ Bieback/Kocher, Juristische Fragen der gesetzlichen Festlegung eines Mindestentgelts durch erleichterte Allgemeinverbindlichkeit oder Verweis auf unterste Tarifentgelte, in: Bieback/Dieterich/Hanau/Kocher/Schäfer, S. $43-102$.

${ }^{52}$ Dieterich, Verfassungsmäßigkeit tarifgestützter Mindestlöhne bei Zeitarbeit, in: Bieback/Dieterich/ Hanau/Kocher/Schäfer, S. 103 - 126; Hanau, Die Einbeziehung der Tarifverträge der Zeitarbeit in das System des Arbeitnehmerentsendegesetzes (AEntG), ebd., S. 127 - 157.

${ }^{53}$ Bieback/Kocher, in: Bieback/Dieterich/Hanau/Kocher/Schäfer, S. 64 f.
} 
Im Hinblick auf die Parteien des Tarifvertrages, dessen Geltungsbereich ausgedehnt werden soll, wird zwar ein Eingriff angenommen ${ }^{54}$, der freilich als wenig gravierend eingeschätzt wird. Immerhin wird seine Rechtfertigung davon abhängig gemacht, dass die Parteien des Tarifvertrages in das Rechtsetzungsverfahren einbezogen werden.

Eine stärkere Eingriffsintensität wird - mit Blick auf den Fall mehrerer einschlägiger Tarifverträge - konstatiert hinsichtlich der Koalitionsfreiheit der Parteien des oder der Tarifverträge, die der Verordnungsgeber bei der Auswahl des auszudehnenden Tarifvertrages nicht auswählt ${ }^{55}$. Zur Rechtfertigung dieser Ausgestaltung der Tarifautonomie wird gefordert, dass dem Verordnungsgeber ein prozedurales Auswahlkriterium vorgegeben werden müsse. Als Auswahlkriterium solle auf die Repräsentativität der konkurrierenden Tarifverträge abgestellt werden; zudem müsse es ein Anhörungsrecht der Parteien übergangener Tarifverträge geben $^{56}$.

Soweit schließlich der durch eine Mindestlohnverordnung zu erstreckende Tarifvertrag nicht allein konkurrierenden Tarifverträgen vorgezogen, sondern diese auch verdrängen soll, gehe es um einen „schweren Eingriff“ ${ }^{\prime 57}$, um einen „,außerordentlich tiefgreifend(en) ${ }^{“ 58}$ Eingriff in die kollektive Koalitionsfreiheit derjenigen Tarifparteien, deren Tarifvertrag das Nachsehen hat. Das prozedurale Auswahlkriterium der Repräsentativität könne den Vorrang der staatlichen Mindestlohnfestsetzung „,nur begrenzt“ rechtfertigen; die Rechtfertigung könne nur gelingen, wenn der Verordnungsgeber überdies durch ausdrückliche gesetzliche Regelung bei der Ausübung seines Ermessens auf eine Prüfung der inhaltlichen Richtigkeit seiner Auswahlentscheidung in dem Sinne verpflichtet werde, dass allein der ausgewählte Tarifvertrag geeignet sein darf, eine Sicherung des Existenzminimums der betroffenen Arbeitnehmer zu gewährleisten; zudem sei ein Anhörungs- und Klagerecht der Parteien des verdrängten Tarifvertrages erforderlich ${ }^{59}$.

\section{Lösung des Konflikts zwischen Dienstleistungsfreiheit und Tarifautonomie aus Sicht des Gesetzgebers}

In der neuen Fassung des AEntG stellt der Gesetzgeber bewusst klar, dass künftig alle in- und ausländischen Arbeitgeber ausnahmslos verpflichtet sind, ihren in Deutschland beschäftigten

\footnotetext{
${ }^{54}$ Bieback/Kocher, in: Bieback/Dieterich/Hanau/Kocher/Schäfer, S. 57 f..

${ }^{55}$ Bieback/Kocher, in: Bieback/Dieterich/Hanau/Kocher/Schäfer, S. 65 f.

${ }^{56}$ Bieback/Kocher, in: Bieback/Dieterich/Hanau/Kocher/Schäfer, S. 67 ff.; Zustimmung zum Kriterium der Repräsentativität bei Dieterich in: in: Bieback/Dieterich/Hanau/Kocher/Schäfer, S. 124.

${ }^{57}$ Bieback/Kocher; in: Bieback/Dieterich/Hanau/Kocher/Schäfer, S. 69.

${ }^{58}$ Dieterich, in: Bieback/Dieterich/Hanau/Kocher/Schäfer, S. 119.

${ }^{59}$ Bieback/Kocher, in: Bieback/Dieterich/Hanau/Kocher/Schäfer, S. 72 - 74.
} 
Arbeitnehmern die tarifvertraglichen Arbeitsbedingungen zu gewähren, die unter das AEntG fallen ${ }^{60}$. Auf diese Weise soll der bereits erwähnten Entscheidung des EuGH in der Rechtssache Portugaia Construcoes Rechnung getragen werden, in der der EuGH, wie bereits erwähnt, eine auf deutsche Unternehmen beschränkte Option, einem allgemeinverbindlichen Mindeststandard zu entgehen, als unzulässige Diskriminierung eingeordnet hatte; zugleich wird in der Gesetzesbegründung auch auf die Rechtsprechung des BAG Bezug genommen ${ }^{61}$, nach der allgemeinverbindliche Tarifverträge im Sinne des AEntG konkurrierenden Tarifverträgen vorgehen.

Überdies werde, wie abschließend betont wird, auf diese Weise sichergestellt, dass es sich bei den tariflichen Mindestregelungen um für allgemeinverbindlich erklärte Tarifverträge im Sinne von Art. 3 Abs. 8 der Entsenderichtlinie handele ${ }^{62}$.

Dass die „Erstreckung von Arbeitsbedingungen bei gleichzeitiger Verdrängung niedriger dotierter Tarifverträge“" auch aus Sicht des Gesetzgebers zu einem verfassungsrechtlichen Problem führt, wird daran deutlich, dass sich die Gesetzesbegründung ausführlich mit den Voraussetzungen beschäftigt, unter denen aus verfassungsrechtlicher Sicht der Erlass einer Mindestlohnverordnung gerechtfertigt werden kann ${ }^{63}$. Der Begründung zufolge ist ein damit verbundene Eingriff in die Tarifautonomie gerechtfertigt, wenn er Gemeinwohlbelangen mit verfassungsrechtlichem Rang dient. Als verfassungsrechtlich legitimierte Regelungszwecke habe das BVerfG die Gewährleistung angemessener Arbeitsbedingungen ${ }^{64}$, die Gewährleistung der finanziellen Stabilität der Systeme der sozialen Sicherung ${ }^{65}$ sowie die Unterstützung der Ordnungsfunktion der Tarifverträge ${ }^{66}$ anerkannt. Diese verfassungsrechtlichen Vorgaben würden durch die in $\S 1$ AEntG genannten Gesetzesziele konkretisiert.

Hinsichtlich der Ausgestaltung des Verfahrens beim Erlass einer Mindestlohnverordnung sieht das neue AEntG für den Fall der Auswahl eines von mehreren konkurrierenden Tarifverträgen Regelungen vor, die in wesentlichen Punkten den von Bieback und Kocher unterbreiteten Vorschlägen entsprechen. Dies gilt zum einen für die Regelung in § 7 Abs. 4 AEntG 2009, nach der das Ministerium den Parteien aller betroffenen Tarifverträge Gelegenheit geben

\footnotetext{
${ }^{60} \mathrm{Vgl}$. hierzu und zum folgenden die Begründung des Regierungsentwurfs BT-Drs. 16/10486, 12.

${ }^{61}$ BAG 9.9.2003 - 9 AZR 478/02 (A); 18.10.2006 - 10 AZR 576/05 - E 120, 1 = NZA 2007, 1111.

62 BT-Drs. 16/10486, 17.

${ }^{63}$ BT-Drs. 16/10486, 15.

${ }^{64}$ Bezugnahme auf BVerfG 24.5.11977 - 2 BvL 11/74 - E 44, 340 betr. den allgemeinverbindlichen Tarifvertrag.

${ }^{65}$ Bezugnahme auf BVerfG 3.4.2001 - 1 BvL 32/97 - E 103, 293 sowie auf BVerfG 20.3.2007 - 1 BvR 1047/05 - NZA 2007, 609 (Nichtannahmebeschluss zu $\S 1$ a AEntG a.F., betr. Art. 12.

${ }^{66}$ Bezugnahme auf BVerfG 20.3.2007 - 1 BvR 1047/05 - NZA 2007, 609 (Nichtannahmebeschluss zu $§ 1$ a AEntG a.F., betr. Art. 12).
} 
muss, zu der geplanten Verordnung Stellung zu nehmen ${ }^{67}$. Dies gilt zum anderen für die Vorgaben des neuen $\S 7$, dass bei der Auswahlentscheidung die Repräsentativität der jeweiligen Tarifverträge zu berücksichtigen (Abs. 2) und dass - im Falle konkurrierender Anträge auf Allgemeinverbindlichkeit - eine besonders sorgfältige und verfassungssensible Abwägung vorzunehmen (Abs. 3) ist ${ }^{68}$. Die Repräsentativität eines Tarifvertrages ist der Entwurfsbegründung zufolge ein Indiz für die Akzeptanz des Inhalts und die erwünschte höhere Richtigkeitsgewähr der tariflichen Regelung, das im Rahmen einer Gesamtabwägung zu berücksichtigen $\operatorname{sei}^{69}$.

Bieback und Kocher hatten schließlich eine ausdrückliche gesetzlicheVerpflichtung des Verordnungsgebers für notwendig gehalten, zu prüfen, ob allein der ausgewählte Tarifvertrag geeignet ist, die Sicherung des Existenzminimums der betroffenen Arbeitnehmer zu gewährleisten ${ }^{70}$. Eine Regelung, die diesem Vorschlag Rechnung tragen würde, findet sich jedoch im neuen Entsenderecht nicht.

\section{Verfassungsrechtliche Kritik am Vorrang tarifgestützter Mindestlöhne}

Das Konzept der Großen Koalition, im neuen AEntG tarifgestützten Mindestlöhne verdrängende Wirkung zuzuerkennen, ist auf vehemente Kritik gestoßen. Insbesondere haben Sodann/Zimmermann ${ }^{71}$ und Thüsing ${ }^{72}$ den entsprechenden Regelungsvorschlag noch während des Gesetzgebungsverfahrens in umfangreichen Beiträgen für verfassungswidrig erklärt ${ }^{73}$. In der partiellen Verdrängung bereits bestehender konkurrierender Tarifverträge und auch in der entsprechenden Sperre, die eine Mindestlohnverordnung für künftige konkurrierende Tarifverträge darstellt, wird ein Eingriff in die von der kollektiven Koalitionsfreiheit geschützte Tarifautonomie gesehen ${ }^{74}$. Dieser Eingriff in das vorbehaltlos gewährleistete Grundrecht wird als so gravierend eingeordnet, dass er nicht gerechtfertigt werden könne. Zur Sicherstellung angemessener Arbeitsbedingungen sei die verdrängende Wirkung dann nicht erforderlich, wenn Tarifvertragsparteien ihrer Aufgabe, solche Arbeitsbedingungen zu vereinbaren, nachgekommen seien. Wenn aber ein konkurrierender Tarifvertrag vorliege, hätten die Tarifver-

\footnotetext{
${ }^{67}$ Dazu Bieback/Kocher, in: Bieback/Dieterich/Hanau/Kocher/Schäfer, S. 69.

${ }^{68}$ Dazu Bieback/Kocher, in: Bieback/Dieterich/Hanau/Kocher/Schäfe, S. 67 f. und 73.

${ }^{69}$ BT-Drs. 16/10486, 16.

${ }^{70}$ Bieback/Kocher, in: Bieback/Dieterich/Hanau/Kocher/Schäfer, S. 73.

${ }^{71}$ Sodan/Zimmermann, Die Beseitigung des Tarifvorrangs gegenüber staatlich festgelegten Mindestarbeitsentgelten auf dem Prüfstand der Koalitionsfreiheit, ZfA 2008, 526; jetzt auch Sodan/Zimmermann, Tarifvorrangige Mindestlöhne versus Koalitionsfreiheit, NJW 2009, 2001.

72 Thüsing, ZfA 2008, 590.

${ }^{73}$ In diesem Sinne auch Willemsen/Sagan, Mindestlohn und Grundgesetz, NZA 2008, S. 1216 - 122.

${ }^{74}$ Sodann/Zimmermann, ZfA 2008, 526, 544- 546 mit 539 ff.; Thüsing, ZfA 2008, 590, 602 f..
} 
tragsparteien diese ihre Aufgabe gerade erfüllt. Auch mit dem Ziel, den Missbrauch einer eventuellen Tarifdispositivität von Mindestlöhnen zu verhindern, sei der Eingriff nicht zu rechtfertigen, denn dies werde schon durch das Erfordernis der Tariffähigkeit der Tarifvertragsparteien gewährleistet ${ }^{75}$. Eine Rechtfertigung des Eingriffs komme nur in Frage, soweit es um die Sicherung eines existenzsichernden Minimallohnes gehe; die neue Regelung ziele jedoch auf ,,angemessene Arbeitsbedingungen“, also auf ein höheres Niveau ${ }^{76}$. Auch das Ziel der Bekämpfung der Arbeitslosigkeit könne die Verdrängung konkurrierender tariflichvertraglicher Lohnregelungen nicht rechfertigen; die Mindestlohnvorgaben seien mit Blick auf dieses Ziel ungeeignet, da sie die Arbeitslosigkeit regelmäßig eher beförderten ${ }^{77}$. Es werde auch nicht, wie als weiteres Ziel angegeben, die Ordnungsfunktion der Tarifverträge unterstützt; diese werde vielmehr durch die Verdrängung konkurrierender Tarifverträge untergraben $^{78}$. Hingewiesen wird überdies darauf, dass eine Kollision mit der Dienstleistungsfreiheit den Gesetzgeber nicht zur Beseitigung des Vorrangs konkurrierender Tarifverträge zwinge. Eine Privilegierung inländischer Unternehmen durch die Option, mittels abweichender Tarifverträge erstreckte Mindestentgeltregelungen zu unterschreiten, lasse sich auch dadurch vermeiden, dass man die nämliche Option auch ausländischen Unternehmen verschaffe ${ }^{79}$.

Thüsing hat ausführlich Kritik am Kriterium der Repräsentativität geübt, das nach dem neuen Recht beim Erlass einer Mindestlohnverordnung vom Verordnungsgeber berücksichtigt werden soll, wenn es darum geht, unter konkurrierenden Tarifverträgen einen auszuwählen, dem zwingende Wirkung verliehen werden soll ${ }^{80}$. Insbesondere hat er ausgeführt, dieses Kriterium führe ,zu dogmatischen Friktionen“, da es zu einer Vermengung inkommensurabler Größen führe; es sei nicht möglich, die Zielvorgaben des AEntG mit dem lediglich operationalen Kriterium der Repräsentativität in Ausgleich zu bringen. Es sei auch nicht zu rechtfertigen, dass dieses Kriterium größere Tarifparteien gegenüber kleineren begünstige.

\section{Verfassungsrechtliche Stellungnahme}

Es lässt sich nicht bestreiten, dass die Verdrängung tariflicher Regelungen durch die Erstreckung eines anderen Tarifvertrages im Wege einer Mindestlohnverordnung gravierend in die grundrechtlich geschützte Tarifautonomie eingreift. Ebensowenig lässt sich allerdings leug-

\footnotetext{
${ }^{75}$ So Thüsing, ZfA 2008, 590, 613;

${ }^{76}$ Sodan/Zimmermann, ZfA 2008, 526, 548 - 550.

77 Thüsing, ZfA 2008, 590, 605 - 608; Sodan/Zimmermann, ZfA 2008, 526, 552- 555.

${ }^{78}$ Sodan/Zimmermann, ZfA 2008, 526, $560 \mathrm{f..}$

${ }^{79}$ Sodan/Zimmermann, ZfA 2008, 526, 573 ff., 578 m.w.N.).

${ }^{80}$ Thüsing, ZfA 2008, 590, $629-639$.
} 
nen, dass sich der Gesetzgeber des AEntG aus europarechtlichen Gründen in einer gewissen Zwickmühle befindet. Die in der Dienstleistungsfreiheit wurzelnde Pflicht zur Gleichbehandlung von Unternehmen aus anderen Mitgliedstaaten erzwingt die gleichmäßige Durchsetzung arbeitsrechtlicher Mindeststandards. Schlupflöcher, die nur für deutsche Unternehmen gangbar sind, sei es auch nur aus tatsächlichen Gründen ${ }^{81}$, müssen geschlossen werden. Will der Gesetzgeber also mit dem - im Ausgangspunkt gerade tariffreundlichen - Instrument tarifgestützter Mindestlöhne eine Mindestlohnpolitik betreiben, bleibt ihm nichts anderes übrig, als diese Mindestlöhne in einem strikten Sinne zwingend auszugestalten. Vor diesem Hintergrund kann auch aus Art. 9 Abs. 3 GG nicht abgeleitet werden, dass eine derartige Regelung a priori nicht in Betracht komme. Verfassungsrechtliche Anforderungen können sich vielmehr allein auf die prozeduralen und sachlichen Vorgaben für die Auswahl des zu erstreckenden Tarifvertrages beziehen. M.E. sollte man insoweit an den Vorschlag von Bieback und Kocher anknüpfen ${ }^{82}$ und vom Verordnungsgeber eine an der Sicherstellung des Existenzminimums orientierte Handhabung von Auswahlentscheidungen verlangen, auch wenn dies vom Gesetzeswortlaut nicht ausdrücklich vorgegeben wird. Der Verordnungsgeber sollte auch einen Tarifvertrag mit vergleichsweise hohem Grad an Repräsentativität ${ }^{83}$ einem konkurrierenden Tarifvertrag nur dann vorziehen dürfen, wenn das im konkurrierenden Tarifvertrag vorgesehene Lohnniveau zur Existenzsicherung nicht ausreicht ${ }^{84}$. Zur Bestimmung des hierfür erforderlichen Maßstabes könnte man auf das im SGB II vorgesehene Leistungsniveau abstellen, u.U. unter Berücksichtigung der dort vorgesehenen Freibeträge ${ }^{85}$.

\section{Mindestlöhne und Hartz-Gesetzgebung}

Es hat sich nach allem gezeigt, dass das AEntG 2009 Ausdruck eines politischen Ansatzes ist, der auf die Durchsetzung von Mindestlöhnen abzielt, die viele Branchen erfassen. Der Hartzgesetzgebung hingegen ging es nicht um die Sicherung von Mindestlöhnen, im Gegenteil. Es

\footnotetext{
${ }^{81}$ Überzeugend Bayreuther, NJW 2009, 2006, 2010.

${ }^{82}$ Nochmals Bieback/Kocher, in: Bieback/Dieterich/Hanau/Kocher/Schäfer, S. 72.

${ }^{83}$ Mit Recht wird darauf hingewiesen, dass die Repräsentativität der Tarifvertragsparteien im Tarifrecht anderer Staaten vielfach eine Rolle spielt; vgl. Bayreuther, NJW 2009, S. 2006 (2008); Gamillscheg, Kollektives Arbeitsrecht I, München 1997, S. 439 ff.; vgl. auch Art. 3 Abs. 8 Ua. 2, 2. Spiegelstrich der Richtlinie 96/71/EG des Europäischen Parlaments und des Rates vom 16.Dezember 1996 über die Entsendung von Arbeitnehmern im Rahmen der Erbringung von Dienstleistungen, ABl. 1997 Nr. L 18 S. 1.

${ }^{84}$ Ähnlich Sittard, NZA 2009, 346, 349.

${ }^{85}$ Diese Möglichkeit wird de lege ferenda im Hinblick auf einen möglichen allgemeinen Mindestlohn diskutiert bei Bispinck/Schulten, Aktuelle Mindestlohndebatte. Branchenlösungen oder gesetzlicher Mindestlohn?, WSIMitt. 2008, 151; diese Autoren errechnen auf dieser Grundlage einen Brutto-Mindestlohn in Höhe von 8,14 Euro pro Stunde; ähnlicher rechtspolitischer Ansatz bei Bayreuther, Gesetzlicher Mindestlohn und sittenwidrige Arbeitsbedingungen, NJW 2007, 2022, 2025.
} 
hatte zwar im Gesetzgebungsverfahren des Zweiten Gesetzes über moderne Dienstleistungen am Arbeitsmarkt einen politischen Vorstoß gegeben, dessen Impetus mit dem Mindestlohngedanken verknüpft war und der eine heftige politische Kontroverse herbeiführte. Der Streit ging um die Zumutbarkeitsregelung (§ 10 SGB II), also um die Frage, auf welche Arbeitsbedingungen sich ein Bezieher von Leistungen der Grundsicherung für Arbeitsuchende einlassen muss, wenn der Leistungsanspruch nicht gefährdet werden soll. Nach dem Entwurf der Fraktionen von SPD und Bündnis 90/Die Grünen war für das Entgelt einer dem Arbeitsuchenden angebotenen Beschäftigung keinerlei Untergrenze vorgesehen ${ }^{86}$. Dieses Konzept stieß auf Protest des DGB und auch bei sechs linken Abgeordneten der SPDBundestagsfraktion, die ihre Zustimmung zu dem Gesetz von der Einfügung einer Mindestlohnklausel abhängig machten ${ }^{87}$. Der Bundestag trug dem Protest zunächst durch Einfügung einer Klausel Rechnung, derzufolge ein Arbeitsangebot dann unzumutbar sein sollte, wenn „für die Arbeit nicht das maßgebliche tarifliche Arbeitsentgelt oder mangels einer tariflichen Regelung das ortsübliche Arbeitentgelt gezahlt wird“" ${ }^{\circ 8}$. Der Bundesrat jedoch verweigerte diesem Vorschlag die Zustimmung. Im Vermittlungsausschuss knüpften die Bundesländer ihre Zustimmung an eine Abkehr vom Konzept des Mindestlohnes in der Zumutbarkeitsregelung. Deshalb gelangte schließlich doch die bereits im Gesetzesentwurf vorgesehene Version der Zumutbarkeitsregelung ins SGB II, nachdem Bundestag und Bundesrat dem Gesetz jeweils mit großer Mehrheit zugestimmt hatten. Vor diesem Hintergrund kann das SGB II nicht als Mindestlohngesetz angesehen werden. Es geht dem Gesetz vielmehr um die sozialrechtliche Gewährleistung eines vom Staat bereitzustellenden Existenzminimums, wobei den solchermaßen gesicherten Personen auch die Annahme von Beschäftigungen im Niedriglohnsegment angesonnen werden können soll.

Dass das SGB II nicht vom Mindestlohn-, sondern vom Niedriglohndenken beherrscht wird, lässt sich überdies an der Entstehungsgeschichte der Regelung über den Erwerbstätigenfreibetrag (§ 30 SGB II) nachweisen. Hinter der partiellen Anrechnungsfreiheit geringer Einkommen steht ebenfalls die Überlegung, auf diese Weise könne dazu beigetragen werden, ,auch in Deutschland einen Niedriglohnsektor wie in Großbritannien und in den USA zu entwickeln. " 89

Aber selbstverständlich ist der Trend zum Mindestlohn nicht ohne Bedeutung für die HartzGesetze, insbesondere nicht für das SGB II, denn Mindestlöhne wirken sich, da Beschäftigun-

\footnotetext{
${ }^{86}$ BT-Drs. 15/1516, 11.

${ }^{87}$ FAZ v. 7.10.2003, S. 14 und v. 14.10.2003, S. 1 f.

${ }^{88} \S 10$ Abs. 1 Nr. 5 SGB II in der Fassung der Ausschussempfehlung, BT-Drs. 15/1728, 174.

89 So der Entwurf eines „Existenzgrundlagengesetzes“ der Fraktion der CDU/CSU, der den späteren $\S 30$ SGB

II (in seiner ursprünglichen Fassung) merklich geprägt hat, BT-Drs. 15/1523, 70.
} 
gen mit rechtswidrig niedrigen Löhnen niemandem angesonnen werden dürfen, auf die $\mathrm{Zu}$ mutbarkeitsschwelle des $\S 10$ SGB II aus. Der Wandel des Entsenderechts bewirkt also zugleich einen deutlichen Wandel im SGB II als einem wesentlichen Teil der Hartzgesetzgebung.

Umgekehrt könnte das SGB II für die Anwendung des AEntG bedeutsam werden, wenn man dem Vorschlag folgt, die Verdrängung konkurrierender Tarifverträge nur dann zuzulassen, wenn diese nicht das Existenzminimum im Sinne des SGB II sicherstellen können. 